\title{
BMJ Open Protocol for a prospective, multicentre registry study of stenting for symptomatic intracranial artery stenosis in China
}

\author{
Yilong Wang, ${ }^{1}$ Zhongrong Miao, ${ }^{2}$ Yongjun Wang, ${ }^{1}$ Xingquan Zhao, ${ }^{1}$ Peiyi Gao, ${ }^{3}$ \\ Liping Liu, ${ }^{1}$ Feng Wang, ${ }^{4}$ Yajie Liu, ${ }^{5}$ Ning Ma, ${ }^{2}$ Ziqi Xu, ${ }^{6}$ Dapeng Mo,, ${ }^{2}$ Feng Gao, ${ }^{2}$ \\ China registry study group
}

To cite: Wang $Y$, Miao Z, Wang $Y$, et al. Protocol for a prospective, multicentre registry study of stenting for symptomatic intracranial artery stenosis in China. BMJ Open 2014;4:e005175. doi:10.1136/bmjopen-2014005175

- Prepublication history and additional material is available. To view please visit the journal (http://dx.doi.org/ 10.1136/bmjopen-2014005175).

Received 11 March 2014

Revised 25 July 2014

Accepted 28 July 2014

\section{CrossMark}

For numbered affiliations see end of article.

Correspondence to Dr Zhongrong Miao: zhongrongm@163.com

\section{ABSTRACT}

Introduction: The SAMMPRIS trial suggested that aggressive treatment was superior to endovascular stenting in patients with severe symptomatic intracranial atherosclerotic stenosis (ICAS) due to high complication rates in patients in the stenting group. Given that $12.2 \%$ patients failed aggressive medical therapy in the SAMMPRIS study, it is imperative to perform a multicentre prospective registry study of stenting for patients with ICAS in China. This study aims to evaluate the safety and efficacy of endovascular stenting for patients with symptomatic intracranial artery stenosis and poor collaterals in China and to identify the characteristics of the population that would benefit the most from endovascular stenting in Chinese patients.

Methods and analysis: This multicentre prospective registry study will involve 20 stroke centres in China, and plans to recruit 300 patients into the registry. Patients with $\geq 70 \%$ stenosis and symptomatic intracranial atherosclerotic disease caused by hypoperfusion combined with poor collaterals who met the inclusion criteria and exclusion criteria would be enrolled for this study. The primary outcome is the target vessel stroke event (including haemorrhagic or ischaemic stroke) or death within 30 days after stenting. The secondary outcomes include the successful recanalisation rate, the incidence of recurrent ischaemic stroke in the territory of the stented artery between 30 days and 1 year postoperatively, the restenosis rate and health-related quality of life.

Ethics and dissemination: The protocol is approved by the ethics committee at the coordinating centre and by the local institutional review board at each participating centre. Findings will be shared with participating hospitals, policymakers and the academic community to promote quality monitoring, quality improvement and the efficient allocation and use of cerebral catheterisation and intracranial artery stenting in China.

Trial registration number: http://www.clinicaltrials. gov (NCT01968122).

\section{INTRODUCTION}

Atherosclerotic intracranial artery stenosis is an important cause of ischaemic stroke. ${ }^{12}$ As shown by the warfarin-aspirin symptomatic intracranial disease (WASID) study, the risk of recurrent ischaemic stroke was still high in patients with intracranial artery stenosis even after aspirin therapy and standard treatment of vascular risk factors. The overall rate of any stroke or death at 1 year was $22 \%$ in WASID for patients with $50-99 \%$ stenosis, ${ }^{3}$ which can cause disability in nearly half of these patients. ${ }^{4}$ In particular, for patients with a high degree of stenosis ( $\geq 70-99 \%)$, the ischaemic stroke recurrence rate at 1 year was $18 \%$. The Wingspan stent system has been widely used to treat patients with symptomatic intracranial atherosclerotic stenosis who have failed conventional therapy. ${ }^{5}$ It has demonstrated efficacy and safety in case series and registries, ${ }^{6-8}$ with a perioperative stroke mortality or postoperative ipsilateral stroke of $9.3-15 \%$ in 6 months to 1 year after endovascular stenting for patients with 50$99 \%$ intracranial artery stenosis, ${ }^{6-8}$ showing a significant decrease in overall stroke recurrence rate when compared with the WASID study. SAMMPRIS compared the efficacy between aggressive medical treatment and endovascular stenting in patients with severe intracranial artery stenosis. ${ }^{9}{ }^{10}$ The results suggested that aggressive medical treatment was superior to endovascular stenting in patients with severe symptomatic intracranial artery stenosis (stenosis $\geq 70-99 \%$ ). Although the results of SAMMPRIS were questioned by many due to various reasons, ${ }^{11-14}$ the observation that $12.2 \%$ patients failed aggressive medical therapy suggested that better therapies need to be developed to improve their outcome. 
Unlike the Western population, the Asian population has a higher prevalence of intracranial artery stenosis, with $26 \%$ of ischaemic strokes resulting from intracranial arterial stenosis as compared with $10 \%$ in Caucasians. ${ }^{15-17}$ A Chinese single-centre registry for intracranial artery stenosis showed that incidences of postoperative stroke within 30 days and ipsilateral stroke between 30 days and 1 year were $6.74 \%$ and $7.3 \%$, respectively, in patients with a stenosis of $\geq 70-99 \%,{ }^{18} 19$ significantly lower than the reports described above, suggesting that endovascular stenting was safe in the Chinese population and that the recurrent stroke risk was reduced significantly in high-volume stenting centres. What is the 'real world' situation of endovascular stenting in China? Multicentre prospective studies in the Chinese population are urgently needed to assess the perioperative efficacy and safety of stenting with different types of stents in the Chinese population, as well as to identify which subgroup of patients would benefit the most from endovascular stenting.

\section{METHOD/DESIGN}

This study is a multicentre prospective single-arm registry study and the protocol is approved by the ethics committee at the coordinating centre and by the local institutional review board at each participating centre. This study is initiated by the investigators, with 20 participating stroke centres (see online supplementary appendix II), and plans to recruit 300 consecutive patients who meet the inclusion and exclusion criteria. After the enrolment, all participants would be evaluated at baseline, day 4, day 30, months 6 , months 9 and year 1 (see online supplementary appendix I). The Wingspan stent system is provided by the Boston Scientific company and the Apollo stent is provided by the MicroPort Company, but they will not participate in data collection, analysis, editing or make decisions about the publication.

This study is sponsored and conducted by the Cerebrovascular Disease Center of Tiantan Hospital in addition to its responsibility for data analysis. An independent Data and Safety Monitoring Board (DSMB) oversees the conduction, safety and efficacy of the study. The study has been registered and the registration number is NCT01968122.

\section{Study status}

The study protocol has been approved by the ethics committee and has started recruitment of patients since January 2014 and plans to end recruitment by January 2015.

\section{PATIENT POPULATION}

Any patient with $\geq 70 \%$ stenosis and symptomatic intracranial atherosclerotic disease caused by hypoperfusion combined with poor collaterals who meet the inclusion and exclusion criteria are eligible (boxes 1 and 2).

\section{Box 1 Inclusion criteria}

1. Patients aged $18-80$ years old.

2. Patients with primary or recurrent symptomatic intracranial arteriosclerosis who failed medical treatment (ie, stroke or transient ischaemic attack within 90 days prior to enrolment, on at least one antithrombotic drug and vascular risk factor control).

3. Stenosis of $\geq 70 \%$ (as measured using the warfarin-aspirin symptomatic intracranial disease method) and a lesion length of $\leq 15 \mathrm{~mm}$ and $\geq 2.0 \mathrm{~mm}$ diameter on digital subtraction angiography (DSA), with normal distal vessel.

4. Hypoperfusion in the territory of the culprit artery as determined on imaging within 2 weeks before the operation, using any one of the following methods:

i. A cerebral blood flow decrease of $\geq 30 \%$ when compared with the perfusion on the contralateral side on perfusion CT.

ii. An American Society of Interventional and Therapeutic Neuroradiology/Society of Interventional Radiology Collateral Flow Grading System score of $<3$ on DSA.

iii. Haemodynamic ischaemic lesion by MRI.

iv. Hypoperfusion by single-photon emission CT.

V. A peak systolic velocity of $\geq 200 \mathrm{~cm} / \mathrm{s}$ on transcranial Doppler examination.

5. Written informed consent available.

\section{TREATMENT PLAN}

\section{Endovascular intervention}

According to the condition of the patients and the operation specifications and experience of the study centres, the investigators decide the means to treat by endovascular stenting. All the patients are treated under general

\section{Box 2 Exclusion criteria}

1. An acute ischaemic stroke within 3 weeks.

2. $>70 \%$ Stenosis in an intracranial artery other than the culprit artery.

3. $>50 \%$ Stenosis of an extracranial carotid or vertebral artery on the ipsilateral side.

4. Perforator strokes based on MRI.

5. Non-atherosclerotic lesion, for example, moyamoya disease, vascular inflammatory disease due to infection, autoimmunity diseases, postirradiation, postpartum state, developmental or genetic abnormalities, for example, fibromuscular dysplasia, sickle-cell anaemia, suspected vasospasm.

6. Potential cardiac embolism as cause.

7. Intracranial haemorrhage within 6 weeks.

8. Concomitant intracranial tumour, aneurysm or arteriovenous malformation.

9. Known contraindications for heparin, aspirin, clopidogrel, anaesthetics or contrast.

10. Haemoglobin $<10 \mathrm{~g} / \mathrm{dL}$, blood platelet count $<100000$, international normalisation ratio $>1.5$, or other uncorrectable coagulopathies.

11. A baseline modified Rankin Score of $\geq 3$.

12. Life expectancy of $<1$ year due to the concomitant illness.

13. Pregnant or lactating women. 
anaesthesia. Device selection by the Wingspan or the Apollo stent depends on arterial access and lesion morphology. From our experience, the Gateway balloon catheter and Wingspan stent system is more suited to patients with tortuous vascular access, especially at the C4 segment, as this system has better flexibility in traversing curvatures. The Apollo stent is more rigid compared with the Gateway-Wingspan system, but it is preferred for patients with smoother access, as the delivery of this balloon-expandable stent does not require exchanging and less procedural time is needed. Hence, for patients with smooth arterial access and a Mori A lesion, or if the lesion is located in the mid-basilar artery and distal M1 segment, the Apollo balloon-mounted stent would be selected. For patients with tortuous arterial access and a Mori B or C lesion, or a lesion with a significant mismatch in the diameter between proximal and distal segment, angioplasty plus self-expanding stent (Gateway balloon plus Wingspan stent system) is preferred. For patients with tortuous arterial access with a Mori A lesion, or small target vessel diameter $<2.5 \mathrm{~mm}$, direct angioplasty with Gateway balloon would be performed with submaximal dilation. If severe dissection or elastic recoil occurred after angioplasty, the Apollo stent (for patients with less tortuous access) or the Wingspan stent (for patients with severe tortuous access or small target vessel) would be implanted.

\section{Aggressive medical treatment}

The regimen for aggressive risk factor control is based on the SAMMPRIS study and the Chinese ischaemic stroke guideline ${ }^{9}$ : aspirin $(100 \mathrm{mg} /$ day $)+$ clopidogrel ( $75 \mathrm{mg}$ /day) for more than 5 days before the operation (a loading dose of $300 \mathrm{mg}$ clopidogrel is given for emergency operations for patients without prior use of clopidogrel); aspirin (100 mg/day)+clopidogrel ( $75 \mathrm{mg} /$ day) for 90 days poststenting; and for vascular risk factor control: aiming for a target systolic blood pressure of $<140 \mathrm{~mm} \mathrm{Hg}$ (or $<130 \mathrm{~mm} \mathrm{Hg}$ in patients with diabetes); low-density lipoprotein $<70 \mathrm{mg} / \mathrm{dL}(1.81 \mathrm{mmol} / \mathrm{L})$ or a decrease by $50 \%$; smoking cessation; lifestyle modification for obesity and sedentary state.

\section{Primary end point}

The primary end point is the target vessel stroke event (including haemorrhagic or ischaemic stroke) or death within 30 days after stenting.

Ischaemic stroke is defined as a new focal neurological deficit of sudden onset, lasting at least $24 \mathrm{~h}$, that is not caused by haemorrhage as shown on CT or MRI of the brain. Ischaemic strokes are classified as in or out of the territory of the symptomatic intracranial artery.

Symptomatic brain haemorrhage is defined as parenchymal, subarachnoid or intraventricular haemorrhage detected by CT or MRI, associated with seizures or new neurological symptoms or signs (headache, change in level of consciousness, or focal neurological deficits) lasting at least $24 \mathrm{~h}$ and is only regarded as a primary end point if it occurs within 30 days after revascularisation.

\section{Secondary end points}

The secondary end points are the successful recanalisation rate immediately after the procedure (stenosis $<50 \%)$; the incidence of recurrent ischaemic stroke in the involved vascular territory between 30 days and 1 year postoperatively; in-stent restenosis within 1 year postoperatively; ischaemic stroke other than the territory of the stented artery between 30 days and 1 year postoperatively; any cerebral parenchymal, subarachnoid or intraventricular haemorrhage between 30 days and 1 year postoperatively; myocardial infarction; major nonstroke haemorrhage (eg, epidural or subdural haemorrhage, or other major organ system haemorrhage); mortality at 30 days; disabling stroke; any stroke or death; any severe adverse events; neurological functional outcome by the National Institutes of Health Stroke Scale (NIHSS); modified Rankin Scale; cognitive functional outcome by the Montreal Cognitive Assessment (MoCA) in 30 days and 1 year; and Quality of Life (EuroQOL five dimensions questionnaire (EQ-5D) scale) in 1 year.

A diagnosis of myocardial infarction would be considered if the patient had any of the following: typical symptoms of chest pain, ECG evidence of ischaemia including new ST segment depression or elevation $>1 \mathrm{~mm}$ in two or more contiguous leads, typical rise and fall of the cardiac enzymes.

For death, efforts are made to obtain relevant records from the hospital, including death certificates, to determine the cause.

\section{STATISTICAL ANALYSIS}

The results would be analysed primarily through the intention-to-treat method and secondarily by the perprotocol analysis. The influence of confounding factors on outcome would be analysed (box 3).

The Mann-Whitney test would be used for ordinal variables, while the Student $t$ test would be used for continuous variables. Subsequent multivariate analysis by logistic regression would be performed to identify independent predictors of the primary outcome.

\section{Sample size}

By referring to the published Wingspan registration study, ${ }^{6-8}$ the incidence of the primary end point is assumed to be $7 \%$. This registration study will enrol about 300 patients.

\section{STRENGTHS AND LIMITATIONS OF THIS STUDY}

This is a prospective clinical registry that will help clarify whether endovascular angioplasty and stenting is a safe and efficacious therapy as an alternative to aggressive medical treatment for patients with symptomatic severe intracranial atherosclerosis and poor collaterals. The 


\section{Box 3 Confounding factors for analysis}

Patient demographics

- Age,

- Gender,

- Nature of the presenting cerebrovascular event (stroke or transient ischaemic attack)

Characteristics of the stenosis and vessels

- Stenosis severity

- Stenosis site

- Mori grading

- Diameter and length of the involved vessels

Treatment factors

- Compliance with medications (in particular with the antiplatelets-aspirin and clopidogrel).

- Resistance to aspirin or clopidogrel (tested by thrombelastography, plasma concentration or pharmacogenomics).

- Types of antihypertensive drugs used

- Statin use and whether the lipid levels achieve target (lowdensity lipoprotein $70 \mathrm{mg} / \mathrm{dL}$ or decrease by $>50 \%$ ).

- Working experience of the operators with the Wingspan or Apollo stent system.

study evaluates a tailored endovascular treatment with currently available devices based on arterial access and lesion morphology for symptomatic severe intracranial atherosclerosis. However, this study has limitations. It focuses on the Chinese population and caution should be used when applying the findings to patients of other ethnicity. The study has inadequate statistical power to assess the impact of the endovascular treatment on major clinical outcomes.

\section{DATA AND SAFETY MONITORING BOARD}

DSMB members are independent of the researchers and the steering committee. DSMB is responsible for assuring that study participants are not exposed to unnecessary risks and that the study is being conducted according to high scientific and ethical standards. The DSMB is responsible for advising early termination of the study in the event of unexpected safety concerns or if treatment differences were apparent at the prespecified interim analyses.

\section{Author affiliations}

${ }^{1}$ Department of Neurology, Beijing Tiantan Hospital, Capital Medical University, Beijing, China

2Department of Interventional Neuroradiology, Beijing Tiantan Hospital, Capital Medical University, Beijing, China

${ }^{3}$ Department of Neuroradiology, Beijing Neurosurgical Institute, Beijing

Tiantan Hospital, Capital Medical University, Beijing, China

${ }^{4}$ Department of Interventional Radiology, The First Affiliated Hospital of Dalian Medical University, Dalian, China

${ }^{5}$ Department of Neurology, ZhuJiang Hospital of Southern Medical University, Guangzhou, China

${ }^{6}$ Department of Neurology, The First Affiliated Hospital of College of Medicine, Zhejiang University, Hangzhou, China

Contributors Study concept and design were conducted by ZM and YW. Collection of data was done by NM, DM, FG and ZX. Analysis and interpretation of data were performed by NM, DM, FG, ZM and LL. Drafting of the manuscript was done by NM, ZX and ZM. Critical revision of the manuscript was performed by ZM. Statistical analysis was performed by NM and LL. Administrative, technical and material support were provided by DM, FG, FW, YL, NM, ZX, DM, FG and China registry study group. Study supervision was conducted by YiW, YoW, PG and ZM.

Funding This study is sponsored and operated by the Cerebrovascular Disease Center of Tiantan Hospital in addition to its responsibility for data analysis. This study is funded by the National Science and Technology Support Program of 'The 12th Five-Year Plan' of the Ministry of Science and Technology, the National Natural Science Foundation of China, the Beijing High-level Personnel Funds, Boston Scientific Corporation and MicroPort Corporation.

Competing interests None.

Patient consent Obtained.

Ethics approval This study has been approved by the ethics committees at all of the 20 sites.

Provenance and peer review Not commissioned; externally peer reviewed.

Open Access This is an Open Access article distributed in accordance with the Creative Commons Attribution Non Commercial (CC BY-NC 4.0) license, which permits others to distribute, remix, adapt, build upon this work noncommercially, and license their derivative works on different terms, provided the original work is properly cited and the use is non-commercial. See: http:// creativecommons.org/licenses/by-nc/4.0/

\section{REFERENCES}

1. Redon J, Olsen MH, Cooper RS, et al. Stroke mortality and trends from 1990 to 2006 in 39 countries from Europe and Central Asia: implications for control of high blood pressure. Eur Heart $J$ 2011;32:1424-31.

2. Wong KS, Li H, Lam WW, et al. Progression of middle cerebral artery occlusive disease and its relationship with further vascular events after stroke. Stroke 2002;33:532-6.

3. Kasner SE, Chimowitz MI, Lynn MJ, et al.; Warfarin Aspirin Symptomatic Intracranial Disease Trial Investigators. Predictors of ischemic stroke in the territory of a symptomatic intracranial arterial stenosis. Circulation 2006;113:555-63.

4. Famakin BM, Chimowitz MI, Lynn MJ, et al. Causes and severity of ischemic stroke in patients with symptomatic intracranial arterial stenosis. Stroke 2009;40:1999-2003.

5. Fiorella D. Endovascular treatment of intracranial stenosis. World Neurosurg 2011;76(Suppl 6):S66-70.

6. Bose A, Hartmann M, Henkes $\mathrm{H}$, et al. A novel, self-expanding, nitinol stent in medically refractory intracranial atherosclerotic stenoses: the Wingspan study. Stroke 2007;38:1531-7.

7. Zaidat OO, Klucznik R, Alexander MJ, et al. NIH Multi-center Wingspan Intracranial Stent Registry Study Group. The NIH registry on use of the Wingspan stent for symptomatic $70-99 \%$ intracranial arterial stenosis. Neurology 2008;70:1518-24.

8. Fiorella DJ, Turk AS, Levy EI, et al. U.S. Wingspan Registry: 12-month follow-up results. Stroke 2011;42:1976-81.

9. Chimowitz MI, Lynn MJ, Derdeyn CP, et al.; SAMMPRIS Trial Investigators. Stenting versus aggressive medical therapy for intracranial arterial stenosis. N Engl J Med 2011;365:993-1003.

10. Chimowitz Ml. The Feinberg award lecture 2013: treatment of intracranial atherosclerosis: learning from the past and planning for the future. Stroke 2013;44:2664-9.

11. Abou-Chebl A, Steinmetz H. Critique of "Stenting versus aggressive medical therapy for intracranial arterial stenosis" by Chimowitz et al in the new England Journal of Medicine. Stroke 2012;43:616-20.

12. Ringer AJ, Khalessi AA, Mocco J, et al.; Endovascular Neurosurgery Research Group (ENRG). Intervention for intracranial atherosclerosis after SAMMPRIS. World Neurosurg 2012;78:409-12.

13. Abou-Chebl A. Intracranial stenting with Wingspan: still awaiting a safe landing. Stroke 2011;42:1809-11.

14. Derdeyn CP, Fiorella D, Lynn MJ, et al.; SAMMPRIS Trial Investigators. Impact of operator and site experience on outcomes after angioplasty and stenting in the SAMMPRIS trial. $J$ Neurointerv Surg 2013;5:528-33.

15. Wu Z, Yao C, Zhao D, et al. Sino-MONICA project: a collaborative study on trends and determinants in cardiovascular diseases in China, Part I: morbidity and mortality monitoring. Circulation 2001;103:462-8. 
16. Wang $\mathrm{Y}$, Liao X, Zhao X, et al.; China National Stroke Registry Investigators. Using recombinant tissue plasminogen activator to treat acute ischemic stroke in China: analysis of the results from the Chinese National Stroke Registry (CNSR). Stroke 2011;42:1658-64.

17. Wityk RJ, Lehman D, Klag M, et al. Race and sex differences in the distribution of cerebral atherosclerosis. Stroke 1996;27:1974-80.
18. Jiang WJ, Yu W, Du B, et al. Outcome of patients with $\geq 70 \%$ symptomatic intracranial stenosis after Wingspan stenting. Stroke 2011;42:1971-5.

19. Miao ZR, Feng L, Li S, et al. Treatment of symptomatic middle cerebral artery stenosis with balloon-mounted stents: long-term follow-up at a single center. Neurosurgery 2009; 64:79-84. 